REPRINT NO. 21

SOME SIMPLE APPLICATIONS OF THE

TRAVELLING SALESMAN PROBLEM

BY :

J.K. LENSTRA

A.H.G. RINNOOY KAN

$\mathrm{RE} / 75 / 21$

This article appeared in operational Research Quarterley Vol. 26, No. 4, p. 717-733 and is reprinted for private circulation.

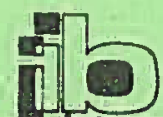

interunhingitaine interfaculteit bedrijfakunde-delft

gneduate school of management defft. the netherlands 


\title{
Some Simple Applications of the Travelling Salesman Problem
}

\author{
J. K. LENSTRA \\ Mathematisch Centrum, Amsterdam \\ and
}

A. H. G. RINNOOY KAN

Graduate School of Management, Delft

\begin{abstract}
The travelling salesman problem arises in many different contexts. In this paper we report on typical applications in computer wiring, vehicle routing, clustering and job-shop scheduling. The formulation as a travelling salesman problem is essentially the simplest way to solve these problems. Most applications originated from real world problems and thus seem to be of particular interest. Illustrated examples are provided with each application.
\end{abstract}

\section{THE TRAVELLING SALESMAN PROBLEM}

\section{Introduction}

IN THIS paper we discuss four apparently unrelated problems that arise in the context of computer wiring, vehicle routing, clustering a data array and job-shop scheduling with no intermediate storage. It turns out that each of these problems can be formulated as a travelling salesman problem (TSP). Three of them originated from real world situations and were not immediately recognized as TSPs; use of TSP algorithms led to better solutions, as will be illustrated below.

Moreover, not only are the four problems special cases of the TSP, but the TSP can conversely be interpreted as a special case of any of these problems. Formulation as a TSP thus is essentially the simplest way to solve them. For the last two problems, their complete equivalence to the TSP is non-trivial.1.2

After introducing the TSP and reviewing the methods for its solution, we will deal with the applications in the remaining sections. Each of the four problems is first described verbally, then formulated as a TSP and finally illustrated by some examples.

\section{Formulation of the TSP}

A salesman wishes to find the shortest route through a number of cities and back home again. This problem is known as the travelling salesman problem and can be stated more formally as follows.

Given a finite set of cities $N$ and a distance matrix $\left(c_{i j}\right)(i, j \in N)$, determine

$$
\min _{\pi} \sum_{i \in N} c_{i \pi(i)}
$$




\section{Operational Research Quarterly Vol. 26 No. 4, i}

where $\pi$ runs over all cyclic permutations of $N ; \pi^{k}(i)$ is the $k$ th city reached by the salesman from city $i$. If $N=\{1, \ldots, n\}$, then an equivalent formulation is

$$
\min _{\nu}\left(\sum_{i=1}^{i=n-1} c_{\nu(i) v(t+1)}+c_{\nu(n) \nu(1)}\right)
$$

where $\nu$ runs over all permutations of $N$; here $\nu(k)$ is the $k$ th city in a saleman's tour. If $G$ denotes the complete directed graph on the vertex set $N$ with a weight $c_{i j}$ for each arc $(i, j)$, then an optimal tour corresponds to a hamiltonian circuit on $G$ (i.e. a circuit passing through each vertex exactly once) of minimum total weight.

If $c_{i j}=c_{j i}$ for all $(i, j)$, the problem is called symmetric, otherwise it is called asymmetric. If $c_{i k} \leqslant c_{i j}+c_{j k}$ for all $(i, j, k)$, the problem is called euclidean.

\section{Solution methods}

References 3, 4 and 5 contain recent surveys of known solution methods.

We can distinguish between optimal and suboptimal algorithms. The first type of algorithm produces solutions that are guaranteed to be optimal but may require inordinate running times; of special interest are the branch-and-bound methods developed by Little et al., Held and Karp ${ }^{7-9}$ and Bellmore and Malone. ${ }^{10}$ Suboptimal algorithms which produce approximate solutions in reasonable times include $\mathrm{Lin}^{11}$ Christofides and Eilon ${ }^{12}$ and $\mathrm{Lin}$ and Kernighan. ${ }^{13}$

In fact, we shall be using the following algorithms:

(a) a branch-and-bound procedure based on Little et al. ${ }^{\circ}$, incorporating an improved branching strategy that allows early pruning of a branch through sufficiently large penalties;

(b) a branch-and-bound procedure based on Held and $\mathrm{Karp}^{8}$ for symmetric TSPs;

(c) a heuristic procedure for generating 3-optimal tours for symmetric TSPs, following the enumeration scheme given by $\operatorname{Lin}^{11}$ with deletion of some superfluous checks for improvement.

Descriptions of these algorithms as well as computational experience and ALGOL 60 procedures can be found in Lenstra. ${ }^{14}$

\section{COMPUTER WIRING}

\section{Problem description}

The following problem arises frequently during the design of computer interfaces at the Institute for Nuclear Physical Research in Amsterdam.

An interface consists of a number of modules, and on each module several pins are located. The position of each module has been determined in advance. A given subset of pins has to be interconnected by wires. In view of possible 


\section{J. K. Lenstra and A. H. G. Rinnooy Kan-Travelling Salesman Problem}

future changes or corrections and of the small size of the pin, at most two wires are to be attached to any pin. In order to avoid signal cross-talk and to improve ease and neatness of wirability, the total wire length has to be minimized.

\section{TSP formulation}

Let $P$ denote the set of pins to be interconnected, $c_{i j}$ the distance between pin $i$ and pin $j$ and $H$ the complete graph on the vertex set $P$ with weights $c_{i j}$ on the arcs.

If any number of wires could be attached to a pin, an optimal wiring would correspond to a minimum spanning tree on $H$, which can be found efficiently by the algorithms of Kruskal ${ }^{15}$ or Prim ${ }^{16}$ and Dijkstra. ${ }^{17}$ However, the degree requirement implies that we have to find a minimum hamiltonian path on $H$ (i.e. a path passing through each vertex exactly once). This problem corresponds to finding a minimum hamiltonian circuit on $G$ with $N=P \cup\{*\}$ and $c_{i *}=c_{* i}=0$ for all $i \in N$. The wiring problem can thus be converted into a symmetric euclidean TSP.

A more difficult problem occurs if the positions of the modules have not been fixed in advance but can be chosen so as to minimize the total wire length for all subsets of pins that have to be interconnected. A review of this placement problem and the associated quadratic assignment problem is given by Hanan and Kurtzberg. ${ }^{18}$

\section{Results}

The procedure that was used originally produced clearly non-optimal wiring schemes like the example with two subsets of pins in Figure 1(a). The size and number of the problems was such that Lin's heuristic had to be used. The 3-optimal results on the example are given in Figure 1(b) (see Visschers and Ten Kate ${ }^{19}$ ).

\section{Problem description}

\section{VEHICLE ROUTING}

In 28 towns in the Dutch province of North-Holland telephone boxes have been installed by the national postal service (PTT). A technical crew has to visit each telephone box once or twice a week to empty the coin box and, if necessary, to replace directories and perform minor repairs. Each working day of at most 445 min begins and ends in the provincial capital Haarlem. The problem is to minimize the number of days in which all telephone boxes can be visited and the total travelling time.

A similar problem arose in the city of Utrecht. Here about 200 mail boxes have to be emptied each day within a period of one hour by trucks operating from the central railway station. The problem is to find the minimum number of trucks able to do this and the associated minimum travelling time. 
Operational Research Quarterly Vol. 26 No. 4, i
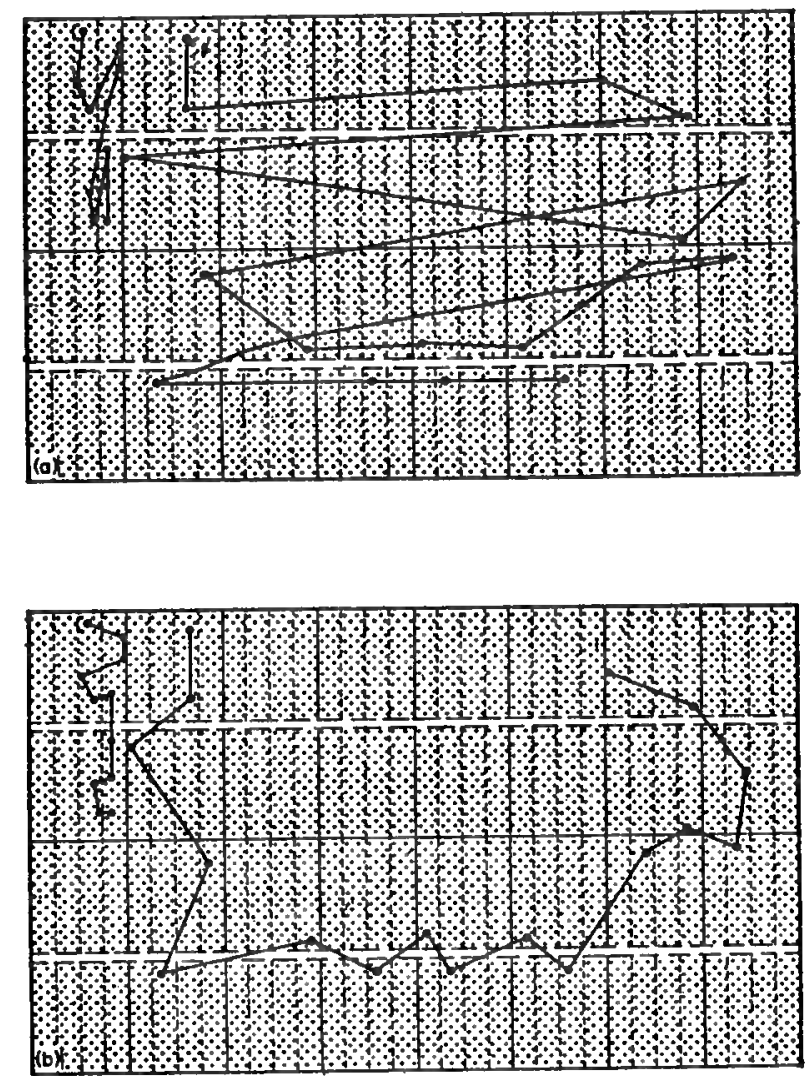

FIG. 1. (a) Wiring without optimization. (b) 3-Optimal wiring.

\section{TSP formulation}

Both problems are types of classical vehicle routing problems (VRP) (see Eilon $e t$ al. ${ }^{5}$ ). They will be denoted by $P 1$ and $P 2$, respectively, and can be characterized more formally as follows:

$n$ cities $i(1 \leqslant i \leqslant n)$ (the customers) are to be visited [P1:28 towns; P2: 200 mail boxes] by $m$ vehicles $[P 1: m$ working days; $P 2: m$ trucks] operating from city * (the depot) $[P 1:$ Haarlem; $P 2$ : central railway station];

the travelling time between cities $i$ and $j$ is $d_{i j}=d_{j i}$ minutes, for

$$
i, j \in\{1, \ldots, n\} \cup\{*\} \text {; }
$$

the time to be spent in city $i$ is $e_{i}$ minutes, for $i \in\{1, \ldots, n\}[P 1: 8 \times$ number of telephone boxes in town $i ; P 2: 1$ ];

the maximum allowable time for any vehicle to complete its route is $f$ minutes [P1: 445; P2: 60]; 


\section{J. K. Lenstra and A. H. G. Rinnooy Kan - Travelling Salesman Problem}

there may be additional constraints [P1: one town (nr. 28, Den Helder) has to be visited twice on different days];

criteria by which solutions are judged are:

$A$, the number of vehicles used;

$B(A)$, the total time used for $A$ vehicles.

If a city has to be visited twice, it is duplicated, appropriate travelling and visiting times are added, and $n$ is increased by one.

[P1: Den Helder is split up into two cities 28 and $29 ; d_{2829}:=\infty ; n:=29$.] We replace the depot (city *) by $m$ artificial depots (cities $n+1, \ldots, n+m$ ) and extend the definition of $\left(d_{i j}\right)$ and $\left(e_{i}\right)$ as follows (cf. Figure 2):

$$
\begin{aligned}
d_{i, n+l}=d_{i \bullet}, & \text { for } 1 \leqslant l \leqslant m ; \\
d_{n+k, j}=d_{* j} & \text { for } 1 \leqslant k \leqslant m ; \\
d_{n+k, n+l}=\lambda & \text { for } 1 \leqslant k, l \leqslant m ; \\
e_{n+k}=0 & \text { for } 1 \leqslant k \leqslant m .
\end{aligned}
$$

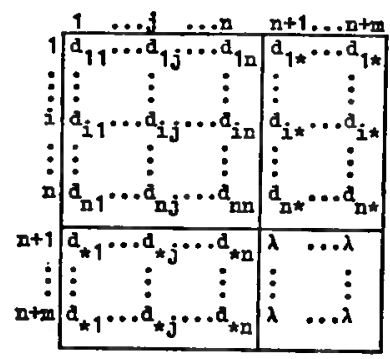

Fio. 2. The matrix $\left(d_{i j}\right)$.

We obtain a symmetric euclidean TSP by defining $N=\{1, \ldots, n+m\}$ and $c_{i j}=\frac{1}{2} e_{i}+d_{i j}+\frac{1}{2} e_{j}$ for all $i, j \in N$. A salesman's tour is feasible for the VRP provided that the time constraint for each vehicle and possible additional constraints are respected. If a TSP solution contains $m-A$ links between artificial depots, then the corresponding VRP solution uses only $A$ vehicles. Adding another vehicle decreases the number of links between artificial depots by one and hence decreases the objective function by $\lambda$. Thus, $-\lambda$ may be interpreted as the cost of a vehicle. We may now consider three possible choices of $\lambda$ :

$\lambda=+\infty$ will lead to $\min _{\pi} B(m)$, i.e. the minimum total time for $m$ vehicles ${ }^{5}$; $\lambda=0$ will lead to $\min _{\pi}\{B(A) \mid 1 \leqslant A \leqslant m\}$, i.e. the minimum total time for any number of vehicles ${ }^{5}$;

$\lambda=-\infty$ will lead to $\min _{\pi} B(\min \{A \mid 1 \leqslant A \leqslant m\})$, i.e. the minimum total time for the minimum number of vehicles. 


\section{Operational Research Quarterly Vol. 26 No. 4, i}

The latter objective is the criterion function for both $P 1$ and $\boldsymbol{P 2}$.

An appropriate method for obtaining good VRP solutions is the following:

(1) Choose an initial tour which satisfies the VRP constraints.

(2) Apply an iterative procedure for improving the tour and check the constraints whenever a possible decrease in tour length occurs.

An interesting variation on this type of problem arises in the context of money collection at post offices. For security reasons, several good routes have to be available. The problem is then equivalent to the moonlighting salesman problem ${ }^{20}$ where $k$ disjoint hamiltonian circuits of minimum total weight are sought. No algorithms for this problem have been proposed so far.

\section{Results}

Figures 3 and 4 illustrate some results, obtained for $P 1$ and $P 2$. In both figures, the links with the depot (*) have not been drawn.

For P1, Lin's heuristic method was used. All 3-optimal solutions obtained require 4 days, representing a 50 per cent decrease with respect to the schedule that was previously used. An example is given in Figure 3(a). Exchanging three links in this solution resulted in the schedule given in Figure 3(b); it involves only 3 days, including however one of $449 \frac{1}{2} \mathrm{~min}$. Computational experience revealed that the heuristic procedure converged much faster with $\lambda=-\infty$ than with $\lambda=0$ (see Kuiper ${ }^{21}$ ).

For $P 2$, a variation on Lin's method was used, whereby only a limited number of promising potential improvements was checked. The number of trucks needed was reduced from ten (Figure $4(a)$ ) to eight (Figures $4(b-d)$ ). In view of the size of the problem, both possibilities $\lambda=0$ and $\lambda=-\infty$ have been run only once; the convergence with $\lambda=-\infty$ was relatively slow.
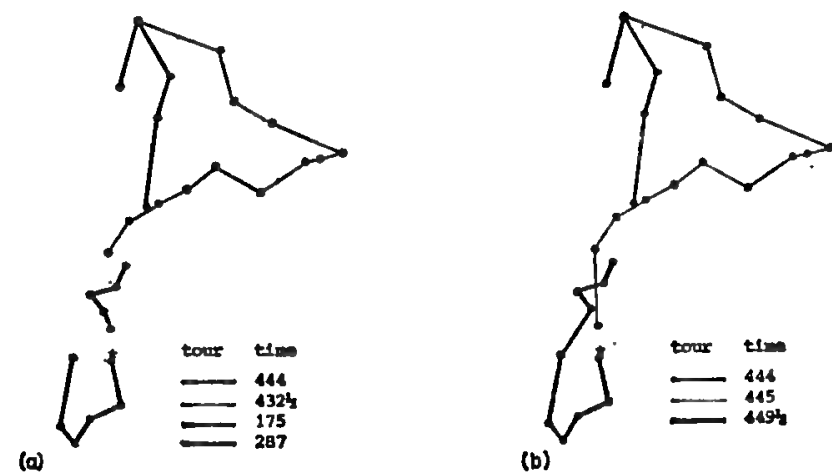

Fio. 3. (a) $P 1$ : 3-optimal solution; $\lambda=-\infty ; B(4)=1338 \frac{1}{2}$. (b) $P 1$ : infeasible solution, obtained by hand from $\mathrm{Fg} .3(\mathrm{a}) ; B(3)=13381$. 

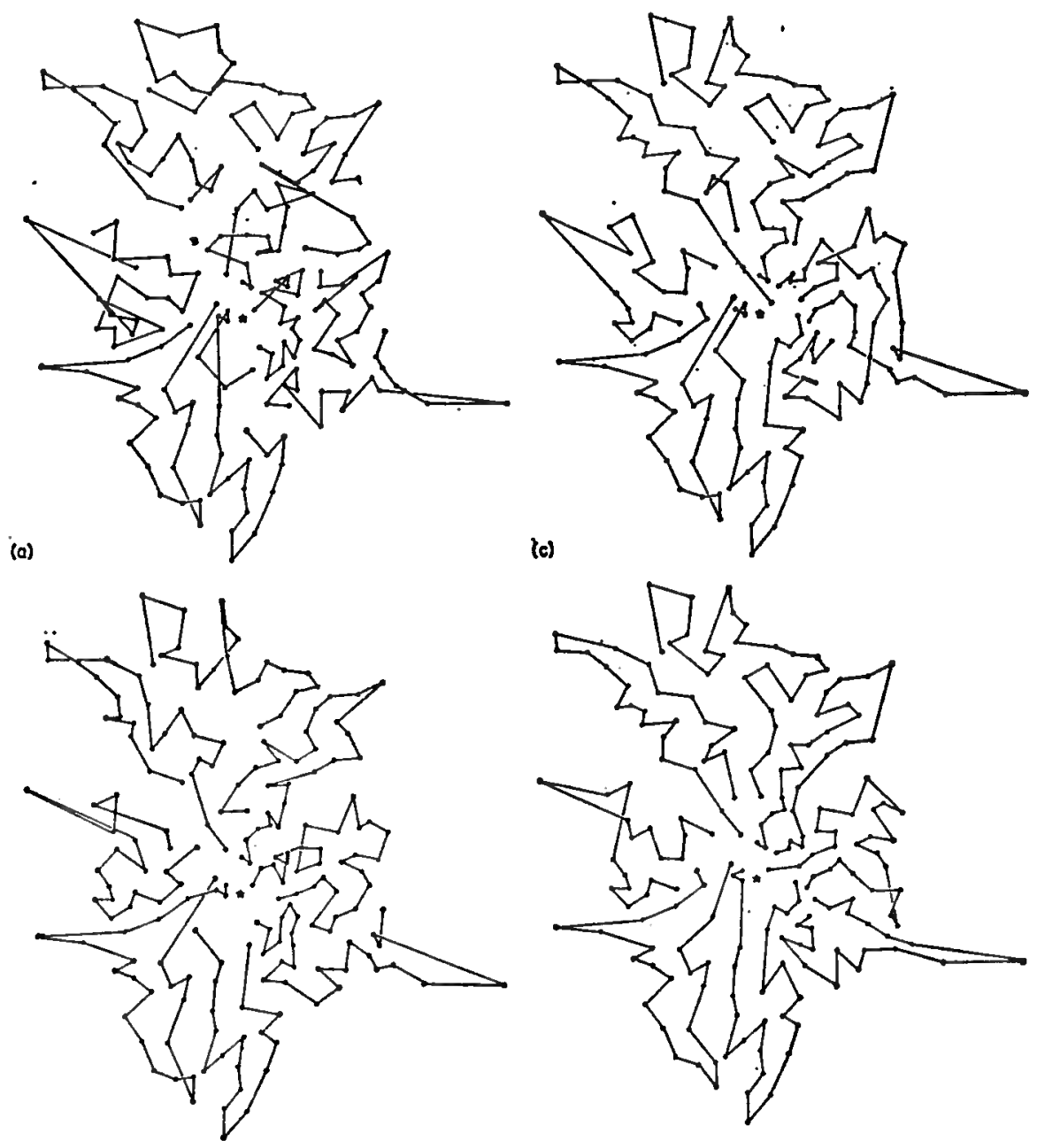

(b)

(d)

FIG. 4. (a) P2: previously used solution; $B(10)=442$. (b) $P 2$ : locally optimal solution, starting from Fig. 4(a); $\lambda=0 ; B(8)=404$. (c) $P 2$ : locally optimal solution, starting from Fig. $4(a) ; \lambda=-\infty ; B(8)=405$. (d) $P 2$ : locally optimal solution, starting from an improvement by hand on Fig. $4(\mathrm{c}) ; \lambda=-\infty ; B(8)=398$. 


\section{Operational Research Quarterly Vol. 26 No. 4, i}

\section{CLUSTERING A DATA ARRAY}

\section{Problem description}

Suppose that a data array $\left(a_{i j}\right)(i \in R, j \in S)$ is given, where $a_{i j}$ measures the strength of the relationship between elements $i \in R$ and $j \in S$. A clustering of the array is obtained by permuting its rows and columns and should identify subsets of $R$ that are strongly related to subsets of $S$.

This situation occurs in widely different contexts. Here we will apply a clustering technique to three examples. In the first one ${ }^{22} R$ is a collection of 24 marketing techniques, $S$ is a collection of 17 marketing applications, $a_{i j}=1$ if technique $i$ has been successfully used for application $j$, and $a_{i j}=0$ otherwise. The second example $e^{29}$ arises in airport design; $R(=S)$ is a set of 27 control variables and $a_{i j}$ measures their interdependence. The third example ${ }^{23}$ deals with an import-export matrix; $R(=S)$ is a set of 50 regions on the Indonesian islands, $a_{i j}=1$ if in 1971 a quantity of at least 50 tons of rice was transported from region $i$ to region $j$, and $a_{i j}=0$ otherwise.

These three examples indicate that the approach is useful for problem decomposition and data reorganization (see McCormick et al. ${ }^{22}$ ).

To convert this problem into an optimization problem, some criterion has to be defined. The proposed measure of effectiveness (ME) is the sum of all products of horizontally or vertically adjacent elements in the array. ${ }^{22}$ Figure 5 shows how this criterion relates to various permutations of a $4 \times 4$ array. The problem is to find permutations of rows and columns of $\left(a_{i j}\right)$ maximizing ME.
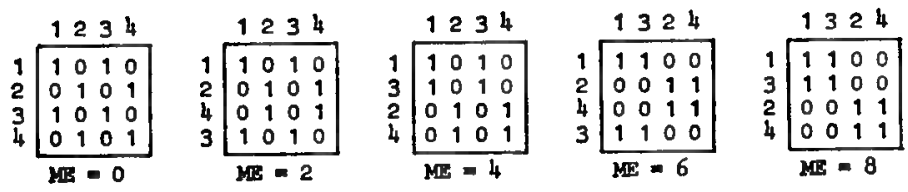

FIG. 5. ME for various permutations of a $4 \times 4$ array.

\section{TSP formulation}

Let $R=\{1, \ldots, r\}$ and $S=\{1, \ldots, s\}$. With the conventions

$$
\begin{gathered}
\rho(0)=\rho(r+1)=\sigma(0)=\sigma(s+1)=*, \\
a_{i \bullet}=a_{* j}=0 \text { for } i \in R, \quad j \in S,
\end{gathered}
$$

the ME, corresponding to permutations $\rho$ of $R$ and $\sigma$ of $S$, is given by

$$
\begin{aligned}
\operatorname{ME}(\rho, \sigma) & =\frac{1}{8} \sum_{i \in R} \sum_{j \in S} a_{\rho(i) \sigma(j)}\left(a_{\rho(i) \sigma(j-1)}+a_{\rho(i) \sigma(j+1)}+a_{\rho(i-1) \sigma(j)}+a_{\rho(i+1) \sigma(j)}\right) \\
& =\sum_{j=0}^{j=8} \sum_{i \in R} a_{i \sigma(j)} a_{i \sigma(j+1)}+\sum_{i=0}^{i=r} \sum_{j \in S} a_{\rho(i) j} a_{\rho(i+1) j} \\
& =\operatorname{ME}(\sigma)+\operatorname{ME}(\rho),
\end{aligned}
$$




\section{J. K. Lenstra and A. H. G. Rinnooy Kan - Travelling Salesman Problem}

so $\operatorname{ME}(\rho, \sigma)$ decomposes into two parts, and its maximization reduces to two separate and similar optimizations, one of $\operatorname{ME}(\sigma)$ for the columns and the other of $\operatorname{ME}(\rho)$ for the rows. McCormick et al. ${ }^{22}$ state that both subproblems may be rewritten as quadratic assignment problems. More precisely, they are symmetric TSPs:

$$
\begin{aligned}
& \text { TSPcol: } \quad N^{\mathrm{col}}=S \cup\{*\}, \quad c_{j k}^{\mathrm{col}}=-\sum_{i \in R} a_{i j} a_{i k} \quad \text { for } j, k \in N^{\mathrm{col}}, \\
& \text { TSProw : } \quad N^{\text {row }}=R \cup\{*\}, \quad c_{h i}^{\text {row }}=-\sum_{j \in S} a_{h j} a_{i j} \text { for } h, i \in N^{\text {row }},
\end{aligned}
$$

for $\operatorname{ME}(\sigma)$ and $\operatorname{ME}(\rho)$, respectively ${ }^{24}$. In general, the clustering problem for a $p$-dimensional array can be stated as $p$ TSPS. It may be attacked by any algorithm for the TSP; in fact, McCormick's ${ }^{22}$ bond energy algorithm (BEA) is a simple suboptimal TSP method which constructs a tour by successively inserting the cities $^{25}$.

If the data array is symmetric (i.e. $a_{i j}=a_{j i}$ for all $i, j$ ), then TSProw and TSPcol are identical and only one optimization needs to be performed (see the airport example).

If the data array is square (i.e. $r=s$ ) but not necessarily symmetric and we want to have equal permutations of rows and columns (i.e. $\rho=\sigma$ ), then one symmetric TSP results:

$$
\text { TSPcow }: \quad N^{\text {cow }}=N^{\text {col }}=N^{\text {row }}, \quad c_{i j}^{\text {cow }}=c_{i j}^{\text {col }}+c_{i j}^{\text {row }} \quad \text { for } i, j \in N^{\text {cow }}
$$

(see the import-export example).

The size of the TSPs might be reduced by assigning identical rows or columns to one single city under the assumption that these rows or columns will be adjacent in at least one optimal solution. This assumption is justified under the conditions expressed by the following theorem.

\section{Theorem}

If $a_{i j} \in\{0,1\}$ for all $i \in R, j \in S$, and $c_{k k l}^{\text {row }}=c_{k l}^{\text {row }}=c_{l l}^{\text {row }}$ for some $k, l \in N^{\text {row }}$, then row $k$ and row $l$ are identical, and adjacent in at least one optimal solution to TSProw.

Proof: We define $S_{i}=\left\{j \mid j \in S, a_{i j}=1\right\}$ for all $i \in N^{\text {row }}$. Since $a_{i j} \in\{0,1\}$ for all $i \in R, j \in S$, we have

$$
c_{i j}^{\text {pow }}=-\left|S_{i} \cap S_{j}\right| \text { for all } i, j \in N^{\mathrm{row}},
$$

and $c_{k k}^{\text {row }}=c_{k l}^{\text {row }}=c_{l l}^{\text {row implies that }} S_{k}=S_{k} \cap S_{l}=S_{l}$. Hence row $k$ and row $l$ are identical:

$$
a_{k j}=a_{l j} \quad \text { for all } j \in S \text {. }
$$

Now consider any permutation $\rho$ of $R$ with $\rho(p)=k, \rho(q)=l,|p-q|>1$. Insert $/$ between $k$ and $\rho(p+1)$. This will not decrease ME( $\rho)$ if

$$
c_{k \rho(p+1)}^{\mathrm{row}}+c_{\rho(Q-1) l}^{\mathrm{row}}+c_{l \rho(q+1)}^{\mathrm{row}} \geqslant c_{k l}^{\mathrm{row}}+c_{l \rho(p+1)}^{\mathrm{row}}+c_{\rho(q-1) \rho(q+1)}^{\mathrm{row}} .
$$


Operational Research Quarterly Vol. 26 No. 4, i

By (1) and (2), this is equivalent to

$$
\left|S_{\rho(q-1)} \cap S_{l}\right|+\left|S_{l} \cap S_{\rho(q+1)}\right| \leqslant\left|S_{l}\right|+\left|S_{\rho(q-1)} \cap S_{\rho(q+1)}\right|,
$$

which is true, since

$$
\begin{aligned}
\mid S_{\rho(q-1)} & \cap S_{l}|+| S_{l} \cap S_{\rho(q+1)} \mid \\
& =\left|S_{l} \cap\left(S_{\rho(q-1)} \cup S_{\rho(q+1)}\right)\right|+\left|S_{l} \cap S_{\rho(q-1)} \cap S_{\rho(q+1)}\right| \\
& \leqslant\left|S_{l}\right|+\left|S_{\rho(q-1)} \cap S_{\rho(q+1)}\right| .
\end{aligned}
$$

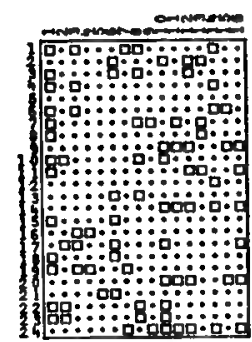

(a)

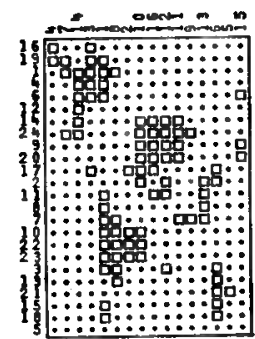

(b)

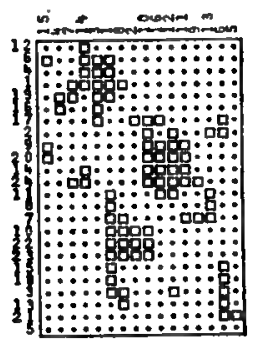

(c)

FIG. 6

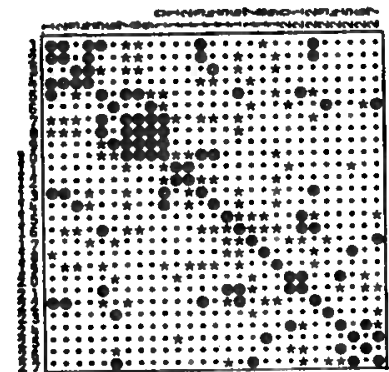

(a)

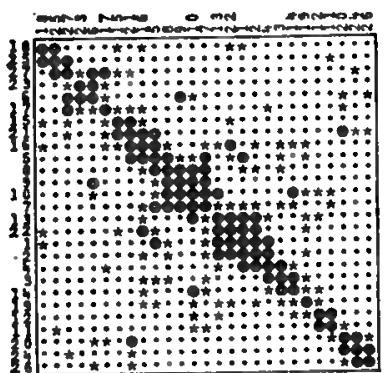

(b)

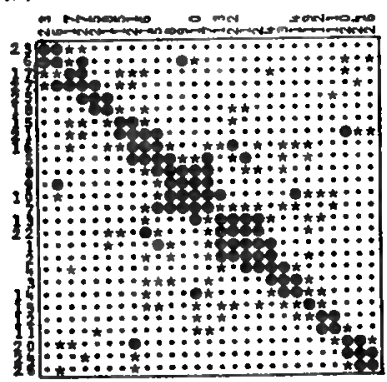

(c)

FIG. 6. Marketing example; $\cdot=0, \square=1$. (a) Initial array; $\mathrm{ME}=39$. (b) BEA clustering; $\mathrm{ME}=97$. (c) Optimal clustering; $\mathrm{ME}=97$.

Fig. 7. Airport example; $\cdot=0, *=1, \otimes=2,0=3$. (a) Initial array; $\mathrm{ME}=592$. (b) $\mathrm{BEA}$ clustering; $\mathrm{ME}=1154$. (c) Optimal clustering; $\mathrm{ME}=1160$. 


\section{J. K. Lenstra and A. H. G. Rinnooy Kan - Travelling Salesman Problem}

Analogous theorems hold for TSP'ol and TSPcow. Definining $R_{j}=\{i \mid i \in R$, $a_{i j}=1$ \} for all $j \in N^{\mathrm{col}}$, we have in the latter case

$$
c_{i j}^{\text {cow }}=-\left|S_{i} \cap S_{j}\right|-\left|R_{i} \cap R_{j}\right| \text { for all } i, j \in N^{\text {cow }},
$$

and we have to show that

$$
\begin{array}{ll}
a_{k j}=a_{l j} & \text { for all } j \in S, \\
a_{i k}=a_{i l} & \text { for all } i \in R .
\end{array}
$$

It follows from (3) and $c_{k k}^{\text {cow }}=c_{k l}^{\text {cow }}=c_{l l}^{\text {cow }}$ that $\left|S_{k}\right|+\left|R_{k}\right|=\left|S_{k} \cap S_{l}\right|+$ $\left|R_{k} \cap R_{l}\right|=\left|S_{l}\right|+\left|R_{l}\right|$. If $\left|S_{k}\right|>\left|S_{k} \cap S_{l}\right|$, then $\left|R_{k}\right|<\left|R_{k} \cap R_{l}\right|$, which is impossible; hence $\left|S_{k}\right|=\left|S_{k} \cap S_{l}\right|=\left|S_{l}\right|$ and $\left|R_{k}\right|=\left|R_{k} \cap R_{l}\right|=\left|R_{l}\right|$, which trivially leads to (4).

These results cannot be generalized to cover the case where $a_{i j}$ can take on other values than 0 or 1 . For example, if $R=\{1,2,3\}$ and $a_{1 j}=a_{2 j}=1, a_{3 j}=2$ for $j \in S$, then the identical rows 1 and 2 are separated by row 3 in the optimal solution.

\section{Results}

The techniques and applications pertaining to the marketing example can be found in McCormick et al. ${ }^{22}$ Figure 6 shows the initial data array, the clustering produced by the $\mathrm{BEA}^{22}$ and a clustering corresponding to optimal solutions of TSPeol and TSProw, found by Little's algorithm after application of the theorem on row identification. It turns out that the BEA clustering is optimal.

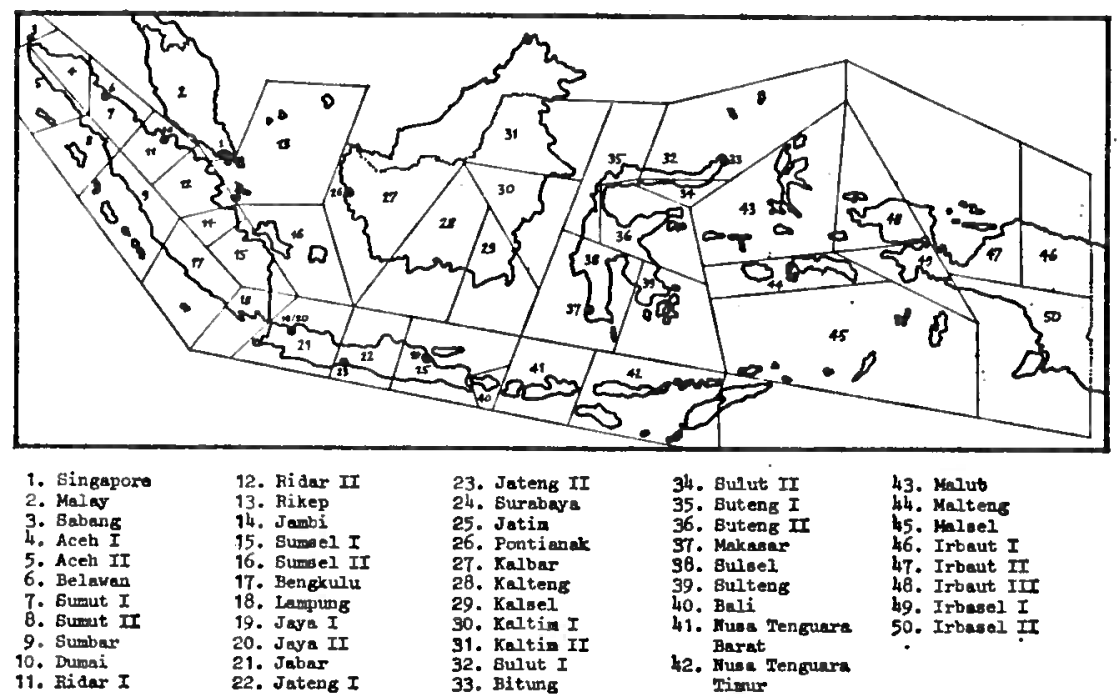

Fig. 8. Import-export example: regions on the Indonesian islands. 


\section{Operational Research Quarterly Vol. 26 No. 4, i}

The control variables in the airport example can be found in McCormick et al..$^{22}$ Figure 7 shows the symmetric initial data array, the BEA clustering ${ }^{22}$ and a clustering corresponding to an optimal solution of $\operatorname{TSP}^{\mathrm{col}}\left(=\mathrm{TSP}^{\circ}\right.$ w), found by Held and Karp's method. The BEA clustering is not optimal and, in fact, not even 3-optimal, since it can be improved by exchanging three links.

The geographical distribution of the regions on the Indonesian islands in the import-export example is given in Figure 8. Figure 9 shows the square but asymmetric initial data array and a clustering corresponding to a 3-optimal solution of TSPoow, found by Lin's heuristic.

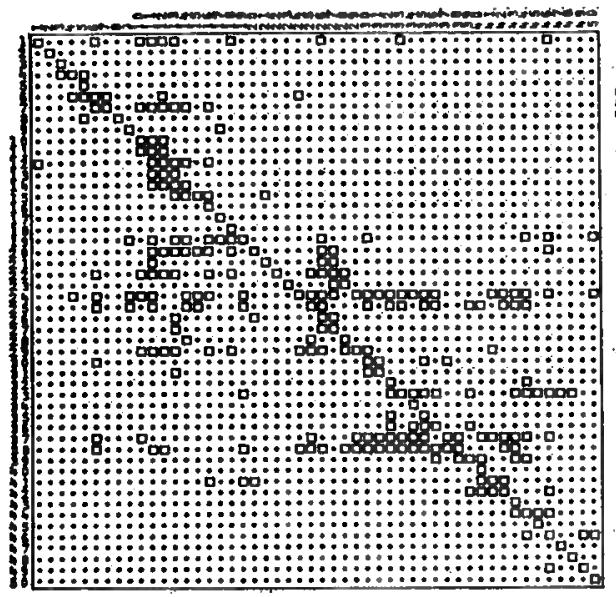

(a)

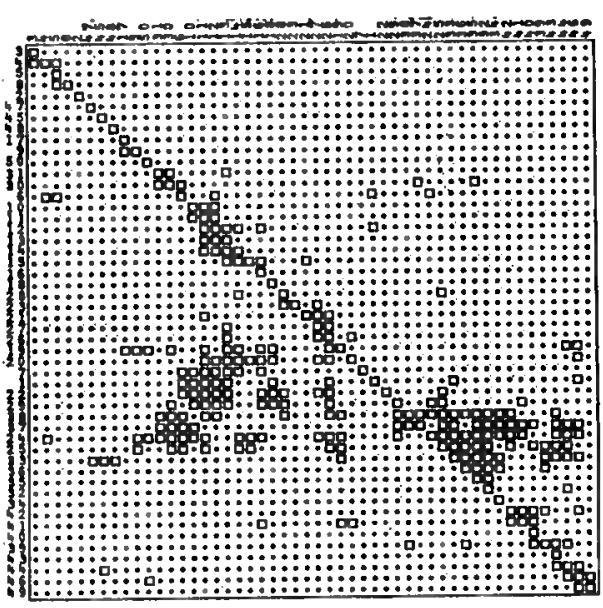

(b)

FIG. 9. Import-export example; $\cdot=0, \square=1$. (a) Initial array; $\mathrm{ME}=223$. (b) 3Optimal clustering subject to $p=\sigma ; \mathrm{ME}=290$.

\section{JOB-SHOP SCHEDULING WITH NO INTERMEDIATE STORAGE}

\section{Problem description}

One of the basic assumptions in most existing theory on machine scheduling is that a job is allowed to wait arbitrarily long before being processed on its next machine. ${ }^{26}$ This assumption is highly unrealistic in some real world situations where intermediate storage space is finite or may even be non-existent. The former situation exists for instance in a computer system where buffer space is limited and costly; the latter situation is met in steel or aluminium rolling where the very high temperature of the metal has to be maintained throughout the production process.

Several researchers ${ }^{27-34}$ have studied the problem of minimizing the total processing time under the restriction of no intermediate storage in a flow-shop, 


\section{J. K. Lenstra and A. H. G. Rinnooy Kan-Travelling Salesman Problem}

where the machine order of each job is identical, which implies that the processing order on each machine will be identical, and simplifies the analysis. Van Deman and Baker ${ }^{35}$ used a different criterion.

Extensions both to non-zero but finite intermediate storage and to different processing orders per machine seem to complicate the situation considerably. We shall restrict our attention to a job-shop where:

(a) the machine order may vary per job;

(b) each job visits each machine at least once;

(c) no passing is permitted, i.e. the processing order is identical on all machines;

(d) no intermediate storage is allowed.

Reddi and Ramamoorthy ${ }^{36}$ consider a more general production process, involving (a), (d) and a "non-subsumption" condition under which lower bounds can be developed. The computation of this lower bound is equivalent to solving a TSP, and the algorithm thus appears to be time-consuming.

\section{TSP formulation}

The job-shop scheduling problem can be described as follows:

$n$ jobs $J_{i}(1 \leqslant i \leqslant n)$ have to be processed on $m$ machines $M_{l}(1 \leqslant l \leqslant m)$;

job $J_{i}(1 \leqslant i \leqslant n)$ consists of $m_{t}$ operations $O_{i k}\left(1 \leqslant k \leqslant m_{t}\right)$;

the machine order of $J_{i}(1 \leqslant i \leqslant n)$ is given by $\mu_{i}=\left(\mu_{i}(1), \ldots, \mu_{i}\left(m_{i}\right)\right)$, i.e. the $k$ th operation $O_{i k}$ of $J_{i}$ has to be performed on $M_{\mu_{i}(k)}$;

the processing time of $O_{i k}\left(1 \leqslant i \leqslant n, 1 \leqslant k \leqslant m_{i}\right)$ is given by $p_{i k}$;

the total processing time has to be minimized under the conditions (a)-(d). We define

$$
\begin{aligned}
k_{i l}^{\prime} & =\min \left\{k \mid \mu_{i}(k)=l, 1 \leqslant k \leqslant m_{i}\right\}, \\
k_{i l}^{\prime \prime} & =\max \left\{k \mid \mu_{i}(k)=l, 1 \leqslant k \leqslant m_{i}\right\}, \\
P_{i} & =\sum_{k=1}^{k=m_{i}} p_{i k}, \\
P_{i l}^{\prime} & =\sum_{k=k_{k l l^{\prime}}}^{k=m_{i}} p_{i k}, \\
P_{i l}^{\prime \prime} & =\sum_{k=1}^{k=k_{i i^{\prime \prime}}} p_{i k} .
\end{aligned}
$$

$O_{i k_{i^{\prime}}}$ and $O_{i k_{i t^{*}}}$ are the first and last operations of $J_{i}$ on $M_{i}$; their existence is ensured by condition (b).

For each pair of jobs $\left(J_{i}, J_{j}\right)$, we will calculate a coefficient $c_{i j}$, representing the minimum difference between the starting times of $O_{i 1}$ and $O_{j 1}$ if $J_{j}$ is scheduled directly after $J_{i}$. By condition (c), $O_{i k_{i l^{\prime}}}$ has to precede $O_{j k_{j i^{\prime}}}$ on $M_{l}$, for $1 \leqslant 1 \leqslant m$. We introduce a directed graph $G_{i j}$ with vertex set $N_{i j}$ and arc set 
Operational Research Quarterly Vol. 26 No. 4, i

$A_{i j}$, defined by

$$
\begin{aligned}
& N_{i j}=\left\{O_{h k} \mid h=i, j, 1 \leqslant k \leqslant m_{h}\right\} ; \\
& A_{i j}=\left\{\left(O_{h k}, O_{h, k+1}\right) \mid h=i, j, 1 \leqslant k \leqslant m_{h}-1\right\} \cup\left\{\left(O_{i k_{k}}, O_{j k_{k j}}\right) \mid 1 \leqslant l \leqslant m\right\} ;
\end{aligned}
$$

a weight $p_{h k}$ is attached to each vertex $O_{h k} \in N_{i j}$. For an example with $m=3$, $\mu_{i}=(2,1,2,3,2)$ and $\mu_{j}=(1,2,3,1)$, the graph $G_{i j}$ is given in Figure 10.

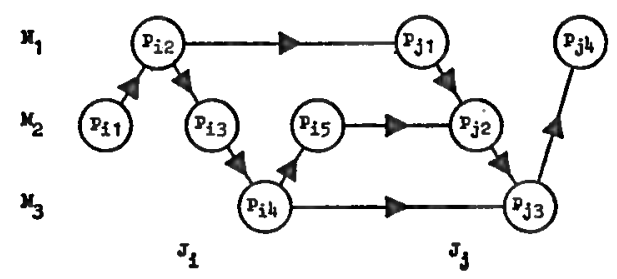

Fig. 10. An example of the graph $G_{i j}$.

As to the path of maximum weight (also called longest or critical path) in $G_{i j}$, it is clear that

$$
\begin{aligned}
& \text { it starts from } O_{i 1} \text { and ends in } O_{j m_{j}} ; \\
& \text { it contains exactly one } \operatorname{arc}\left(O_{i k_{k}}, O_{j k_{j l} l^{\prime}}\right) \text {. }
\end{aligned}
$$

Condition (d) implies that $c_{i j}$ is equal to the latest possible starting time of $O_{j 1}$ in $G_{i j}$ if $O_{i 1}$ starts at time zero and $O_{j m}$ finishes as early as possible. It follows from (5) and (6) that

$$
c_{i j}=\max _{i}\left(P_{i 1}^{n}+P_{j i}^{\prime}\right)-P_{j} .
$$

The minimum total processing time is now given by

$$
\min _{\nu}\left(\sum_{i=1}^{i=n-1} c_{\nu(i) \nu(i+1)}+P_{\nu(n)}\right) \text {, }
$$

where $v$ runs over all permutations of $\{1, \ldots, n\} ; \nu(i)$ is the $i$ th job in a processing schedule.

We add a job $J_{\bullet}$ with $m_{\bullet}=m, \mu_{\bullet}(k)=k$ and $p_{\bullet k}=0$ for $1 \leqslant k \leqslant m$, representing beginning and end of a schedule. According to (7), its coefficients are given by $c_{* i}=0, c_{i *}=P_{i}$ for $1 \leqslant i \leqslant n$. Determination of (8) now corresponds to solving a TSP with $N=\{*\} \cup\{1, \ldots, n\}$ and $\left(c_{i j}\right)$ defined by (7).

This TSP is asymmetric and euclidean. To prove the latter assertion we have to show that $c_{i j}+c_{j k} \geqslant c_{i k}$ for any $i, j, k \in N$, or, equivalently, that

$$
\max _{l}\left(P_{j l}^{n}+P_{j l}^{\prime}\right)+\max _{l}\left(P_{j l}^{n}+P_{k l}^{\prime}\right) \geqslant \max _{1}\left(P_{i l}^{n}+P_{k l}^{\prime}\right)+P_{j} \text {. }
$$




\section{J. K. Lenstra and A. H. G. Rinnooy Kan-Travelling Salesman Problem}

This is true, since for any $l \in\{1, \ldots, m\}$

$$
\left(P_{i l}^{n}+P_{j l}^{\prime}\right)+\left(P_{j l}^{n}+P_{k l}^{\prime}\right) \geqslant\left(P_{u l}^{\prime}+P_{k \nu}^{\prime}\right)+P_{j}
$$

We make two final remarks on this TSP formulation.

Remark 1. In a flow-shop we know that $\mu_{i}=(1,2, \ldots, m)$ for $1 \leqslant i \leqslant m$, and (7) simplifies to $c_{i j}=\max _{l}\left(P_{i l}^{n}-P_{j, l-1}^{n}\right)$ (note that $\left.P_{j 0}^{n}=0\right) .27,28,33$

Remark 2. So far, distances have been defined as differences between the starting times of the first operations of jobs. More generally, one might arbitrarily select any two operations $O_{i k_{i}^{*}}$ and $O_{i k_{i} * *}$ for each job $J_{i}$ and define $c_{i j}$ as the minimum difference between the starting times of $O_{i k_{i}^{*}}$ and $O_{j k_{j} * *}$ if $J_{i}$ precedes $J_{f}$ directly. This will lead to modifications in (7) and (8), but to an equivalent TSP. ${ }^{31,32}$

\section{Results}

To illustrate the consequences of the no intermediate storage condition, we solved three job-shop scheduling problems ${ }^{37}$ under this restriction, using Little's TSP algorithm. In Table 1 the solution values are compared with the lengths of the schedules when infinite intermediate storage is allowed. Figure 11 illustrates the optimal schedules for one of these problems; the unrestricted schedule was found by a method of Florian et al. ${ }^{38}$ In general, the conditions of no intermediate storage and no passing can be expected to lead to large amounts of idle time on the machines.

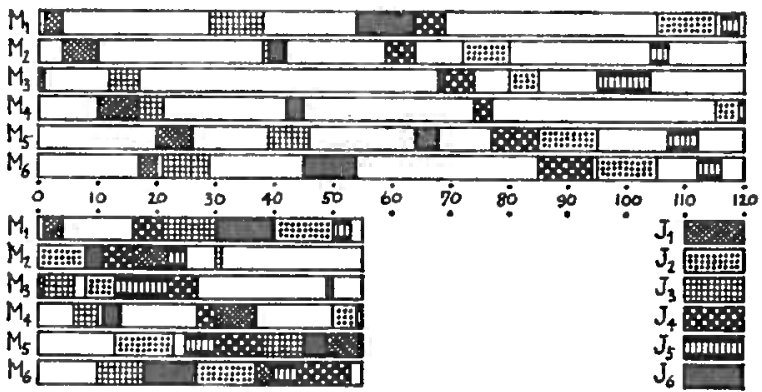

FIG. 11. Optimal schedules for a $6 \times 6$ problem without and with intermediate storage.

TABle 1. EFFECT OF NO INTERMediate STORAGE

\begin{tabular}{cccc}
\hline $\begin{array}{c}\text { Number } \\
\text { of } \\
\text { jobs }\end{array}$ & $\begin{array}{c}\text { Number } \\
\text { of } \\
\text { machines }\end{array}$ & $\begin{array}{c}\text { Value without } \\
\text { intermediate } \\
\text { storage }\end{array}$ & $\begin{array}{c}\text { Value with } \\
\text { intermediate } \\
\text { storage }\end{array}$ \\
\hline 6 & 6 & 120 & 55 \\
10 & 10 & 2433 & $972^{*}$ \\
20 & 5 & 2132 & 1165 \\
\hline
\end{tabular}

* Indicates that the optimality has not been proved. 


\section{Operational Research Quarterly Vol. 26 No. 4, i}

\section{ACKNOWLEDGEMENTS}

We would like to thank B. J. Lageweg (Mathematisch Centrum) for useful comments and programming advice, J. Visschers and P. ten Kate (Instituut voor Kernphysisch Onderzoek) and J. Berendse and J. H. Kuiper (PTT) for implementing the applications to computer wiring and vehicle routing respectively, and $A$. W. Roes and T. J. Wansbeek (Instituut voor Verkeers- en Vervoerseconomie) for making available the data for the import-export example.

\section{REFERENCES}

1 J. K. Lenstra and A. H. G. RINnOoY KAN (1974) Some simple applications of the travelling salesman problem. Report BW 38, Mathematisch Centrum, Amsterdam; Working Paper WP/74/12, Graduate School of Management, Delft.

' P. Brucker, J. K. Lenstra and A. H. G. Rinnooy KAN (1975) Complexity of machine scheduling problems. Report BW 43, Mathematisch Centrum, Amsterdam.

s M. Bellmore and G. L. Nemhauser (1968) The traveling salesman problem: a survey. Ops Res. 16, 538.

A. M. IsAaC and E. Turban (1969) Some comments on the traveling salesman problem. Ops Res. 17, 543.

S S. Enlon, C. D. T. WATSON-GANDY and N. Christofmes (1971) Distribution Management: Mathematical Modelling and Practical Analysis. Griffin, London.

- J. D. C. Litrte, K. G. Murty, D. W. Sweeney and C. Karel (1963) An algorithm for the traveling salesman problem. Ops Res. 11, 972.

7 M. HeLD and R. M. KARP (1970) The traveling-salesman problem and minimum spanning trees. Ops Res. 18, 1138.

- M. HeLd and R. M. KARP (1971) The traveling-salesman problem and minimum spanning trees: part II. Math. Progr. 1, 6.

- K. Helbig Hansen and J. KRaRup (1974) Improvements of the Held-Karp algorithm for the symmetric traveling-salesman problem. Math. Progr. 7, 87.

$10 \mathrm{M}$. Bellmore and J. C. Malone (1971) Pathology of traveling-salesman subtour elimination algorithms. Ops Res. 19, 278, 1766.

11 S. LiN (1965) Computer solutions of the traveling salesman problem. Bell Syst. Tech. $J$. 44, 2245.

12 N. Christofides and S. EILoN (1972) Algorithms for large-scale travelling salesman problems. Opl Res. $Q .23,511$.

13 S. LIN and B. W. Kernighan (1973) An effective heuristic algorithm for the travelingsalesman problem. Ops Res. 21, 498.

14 J. K. LENSTRA (1972) Branch-and-bound algorithmen voor het handelsreizigersprobleem. Report BN 16, Mathematisch Centrum, Amsterdam.

15 J. B. KRuskal (1956) On the shortest spanning subtree of a graph and the travelingsalesman problem. Proc. Am. Math. Soc. 2, 48.

16 R. C. PRIM (1957) Shortest connection networks and some generalizations. Bell Syst. Tech. J. 36, 1389.

17 E. W. Duskstra (1959) A note on two problems in connexion with graphs. Numer. Math. $1,269$.

18 M. Hanan and J. M. Kurtzberg (1972) A review of the placement and quadratic assignment problems. SIAM Rev. 14, 324.

10 J. Visschers and P. TEN KATE (1973) BAKALG, een bedradingsprogramma met optimalisatie van draadlengte. Report SO-1, Instituut voor Kernphysisch Onderzoek, Amsterdam.

20 J. KRARUP (1974) Private communication.

21 J. H. KUIPER (1973) "Hoe een PTT-er handelsreiziger werd"-een routing probleem. Unpublished report.

22 W. T. MCCormick, JR., P. J. Schwertzer and T. W. White (1972) Problem decomposition and data reorganization by a clustering technique. Ops Res. 20, 993.

28 A. W. RoEs (1973) Enige methoden ter verkrijging van een routeschema voor de interinsulaire scheepvaart in Indonesië. Unpublished report. 


\section{J. K. Lenstra and A. H. G. Rinnooy Kan - Travelling Salesman Problem}

24 J. K. Lenstra (1974) Clustering a data array and the traveling-salesman problem. Ops Res. 22, 413.

25 H. Müller-Merbach (1970) Optimale Reihenfolgen. Springer, Berlin.

${ }^{26}$ A. H. G. RINNOOY KAN (1973) The machine scheduling problem. Report BW 27, Mathematisch Centrum, Amsterdam; Report R/73/4, Graduate School of Management, Delft.

27 J. PieHLer (1960) Ein Beitrag zum Reihenfolgeproblem. Unternehmensforschung 4, 138.

${ }^{28}$ S. S. REDDI and C. V. RAMAMOORTHY (1972) On the flow-shop sequencing problem with no wait in process. Opl Res. Q. 23, 323.

29 D. A. WISMER (1972) Solution of the flowshop-scheduling problem with no intermediate queues. Ops Res. 20, 689.

${ }^{30}$ G. Liesegang and M. Rüger (1972) Letter. Opl Res. Q. 23, 591.

31 S. K. GoYal (1973) A note on the paper: On the flow-shop sequencing problem with no wait in process. Opl Res. $Q .24,130$.

32 S. S. Reddi and C. V. RamamoOrthy (1973) Reply to Dr. Goyal's comments. Opl Res. $Q$. 24, 133.

s3 J. Grabowski and M. M. Syslo (1973) On some machine sequencing problems (I). Zastos. Mat. 13, 339.

4. M. Syslo (1974) On some machine sequencing problems (II). Zastos. Mat. 14, 93.

35 J. M. VAN DEMAN and K. R. BAKER (1974) Minimizing mean flowtime in the flow shop with no intermediate queues. AlIE Trans. 6, 28.

${ }^{30} \mathrm{~S}$. S. ReDdi and C. V. Ramamoorthy (1973) A scheduling problem. Opl Res. Q. $24,441$.

37 J. F. MUTH and G. L. Thompson (Eds.) (1963) Industrial Scheduling. Prentice-Hall, Englewood Cliffs, N.J.

88 M. Florian, P. Trepant and G. McMahon (1971) An implicit enumeration algorithm for the machine sequencing problem. Mgmt Sci. 17, B782. 